\title{
Resistencia compresiva en premolares con tratamientos de conductos, restaurados con poste colado y fibra de vidrio anatomizado con distintos niveles de remanente dentario
}

\begin{abstract}
Evaluation of compressive strength in premolars with endodontically treated, restored with metal post
\end{abstract} and fiberglass post with different levels of ferrule

Marcia Vidalón ${ }^{1, a}$, Gustavo Huertas ${ }^{1, b}$

\section{RESUMEN}

Objetivo: Comparar la resistencia compresiva en premolares que presentan tratamiento de conductos con postes colados y fibra de vidrio anatomizados, en diferentes niveles de remanente dentario. Material y Métodos: Conformado por 48 premolares uniradiculares, divididos aleatoriamente en 6 grupos de 8 especímenes cada grupo: Grupo 1: Poste metálico con remanente $0 \mathrm{~mm}$, Grupo 2: Poste metálico con remanente $1 \mathrm{~mm}$, Grupo 3: Poste metálico con remanente $2 \mathrm{~mm}$, Grupo 4: Postes de fibra de vidrio anatomizados con remanente 0 $\mathrm{mm}$, Grupo 5: Postes de fibra de vidrio anatomizados con remanente $1 \mathrm{~mm}$, Grupo 6: Postes de fibra de vidrio anatomizados con remanente $2 \mathrm{~mm}$. El análisis fue comparado por ANOVA y comparaciones múltiples de Tukey a un nivel de significancia de 0.05 . Resultados: Se encontraron diferencias significativas en la resistencia compresiva de premolares con postes metálicos y fibra de vidrio anatomizado en diferentes niveles de remanente dentario. Los grupos con mayor nivel de remanente fueron de $2 \mathrm{~mm}$, el grupo de poste metálico, obtuvo un valor de 885,74 $( \pm 104,10)$ y el grupo de poste de fibra de vidrio anatomizado, un valor de 962,19 $( \pm 104,10)$; $(\mathrm{p}=0,005)$. Conclusiones: El nivel de remanente en postes metálicos y postes de fibra de vidrio anatomizados, influye en la resistencia compresiva, los remanentes $2 \mathrm{~mm}$ restaurados con poste de fibra de vidrio anatomizado y metálico; presentaron mayores valores de resistencia compresiva a diferencia de los de $0 \mathrm{~mm}$ y $1 \mathrm{~mm}$.

PALABRAS CLAVE: Módulo de elasticidad, diente depulpado, endodoncia, fuerza compresiva. 


\section{ABSTRACT}

Objective: To compare the compressive strength in premolars with root canal treatment, restored with metal post and customized fiberglass post, at different levels of ferrule. Material and Methods: It was made up of 48 uniradicular premolars extracted by orthodontic treatment, with similar characteristics, randomly divided into 6 groups of 8 specimens each group: Group 1: Metal post with no ferrule, Group 2: Metal post with 1mm ferrule, Group 3: Metal post with $2 \mathrm{~mm}$ ferrule, Group 4: Customized fiberglass post with no ferrule, Group 5: Customized fiberglass post with ferrule $1 \mathrm{~mm}$, Group 6: Customized fiberglass post with a $2 \mathrm{~mm}$ ferrule. The analysis was compared using ANOVA test and Tukey's multiple comparisons with a significance level of 0.05 . Results: Significant differences were found in the compressive strength of premolars restored with metal post and customized fiberglass post at the different levels of ferrule. The group with ferrule of $2 \mathrm{~mm}$ restored with a metal post, obtained an average value of $885.74( \pm 104.10)$ and with a customized fiberglass post, an average value of $962.19( \pm 104.10)$, being the highest value found in these comparisons; $(p=0.005)$. Conclusions: The level of ferrule in metal post and customized fiberglass post, contributes to compressive strength, $2 \mathrm{~mm}$ ferrule restored with metal post and customized fiberglass post; presented higher values of compressive strength unlike those of $0 \mathrm{~mm}$ and $1 \mathrm{~mm}$.

KEYWORDS: Elastic modulus, tooth nonvital, endodontics, compressive strength.

\section{INTRODUCCIÓN}

La fractura de los dientes es un problema importante que se ha descrito en la odontología, es considerada como la tercera causa más común que tiene como consecuencia perder piezas dentarias, después de la caries dental y la enfermedad periodontal $(1,2,3)$. Los materiales dentales han evolucionado rápidamente en los últimos años, la demanda actual de restauraciones estéticas por parte del paciente obliga a los clínicos a actualizarse constantemente para conocer las diferentes opciones y tomar una adecuada decisión al usar el material correcto $(4,5,6)$. Los dientes con tratamientos de conductos presentan características específicas que deben ser consideradas al momento de planificar la restauración final, en estos casos, existe una pérdida adicional de estructura dentaria la cual es debido al acceso endodóntico, remoción del tejido pulpar y la conformación y preparación de los conductos radiculares $(7,8,9)$. Existen diversas técnicas para rehabilitar dientes con tratamientos de conductos, cuyas indicaciones pueden variar de acuerdo a la cantidad de remanente coronario, soporte óseo, posición del diente en la arcada, la estética, tipo de material utilizado para su rehabilitación, entre otros $(10,11,12)$. Es de suma importancia preservar un adecuado remanente coronario el cual permite la longevidad de la restauración y protección de la integridad de la raíz $(13,14,15)$. Actualmente, se sabe que los postes no sirven para reforzar o fortalecer los dientes con tratamiento de conductos, éstos proveen retención para las restauraciones definitivas $(16,17,18)$. Antes de efectuar cualquier procedimiento dental, debemos examinar al paciente para establecer un diagnóstico correcto con la finalidad de determinar un plan de tratamiento adecuado $(19,20,21)$.

El objetivo de este artículo fue comparar la resistencia compresiva en premolares, los cuales fueron restaurados con postes metálicos y de fibra de vidrio anatomizados en 3 diferentes niveles de remanente coronario.

\section{MATERIAL Y MÉTODOS}

\section{Diseño del estudio:}

El método de esta investigación fue de tipo experimental. El grupo de estudio se obtuvo mediante la fórmula muestral comparando dos medias, estuvo conformado por 48 premolares uniradiculares extraídos por tratamientos de ortodoncia (24 premolares por cada grupo, 8 premolares por cada nivel de remanente coronario), previo consentimiento informado firmado por los pacientes, los premolares presentaron iguales características, los cuales fueron divididos aleatoriamente en 6 grupos de 8 especímenes por cada grupo: Grupo 1: Poste metálico 
con un remanente dentario $0 \mathrm{~mm}$, Grupo 2: Poste metálico con un remanente dentario $1 \mathrm{~mm}$, Grupo 3: Poste metálico con un remanente dentario $2 \mathrm{~mm}$, Grupo 4: Poste de fibra de vidrio anatomizados con un remanente dentario $0 \mathrm{~mm}$, Grupo 5: Poste de fibra de vidrio anatomizados con un remanente dentario 1 $\mathrm{mm}$, Grupo 6: Poste de fibra de vidrio anatomizados con un remanente dentario $2 \mathrm{~mm}$.

Se realizó un estudio piloto con el objetivo de estandarizar los métodos utilizados en el presente estudio. Los datos de este estudio permitieron establecer el tamaño muestral para la siguiente investigación.

\section{Tratamiento de conductos de los especímenes}

Los dientes seleccionados para el estudio, fueron sometidos a un tratamiento de conductos, realizado por un especialista de endodoncia; la longitud de trabajo se estableció a $1 \mathrm{~mm}$ del foramen apical, la preparación del conducto se complementó utilizando la técnica crowndown con rotatorio niquel-titanio (Protaper, Dentsply Maillefer, Suiza). Los dientes fueron instrumentados hasta el tamaño de lima \#40 marca Maillefer, para su obturación los canales radiculares se secaron con aire comprimido y puntas de papel, se utilizaron conos de gutapercha número 40 utilizando la técnica de compactación lateral. El acceso endodóntico se selló con material de obturación temporal Coltosol (Coltene, Suiza). Las muestras se almacenaron durante una semana en agua destilada.

\section{Procedimiento de los postes}

Para la preparación de los remanentes coronarios, las coronas anatómicas fueron cortadas con un disco de corte diamantado (ISO $\mathrm{N}^{\mathrm{o}}$ 016, Shofu, Tokio, Japón) (figura 1 (A)); de la siguiente manera, Grupo 1 y 4: $0 \mathrm{~mm}$ a la unión cemento-esmalte (figura 1 (B)), Grupo 2 y 5: $1 \mathrm{~mm}$ a la unión cemento-esmalte (figura 1 (C)), Grupo 3 y 6: $2 \mathrm{~mm}$ a la unión cemento-esmalte (figura 1 (D)). Los márgenes fueron de $1 \mathrm{~mm}$ de ancho con una configuración de hombro redondeado.

Se debe tener en cuenta que el diámetro del poste debe ser $1 / 3$ del diámetro radicular total, debemos dejar mínimo $3 \mathrm{~mm}$ de gutapercha a nivel apical, con la finalidad de no comprometer el sellado apical durante la desobturación. (12,19,20,21), utilizando fresas Gates y Pesso $N^{o} 1, \mathrm{~N}^{\mathrm{2}} 2$ y $\mathrm{N}^{\mathrm{o}} 3$ de forma intermitente.

\section{Poste metálico \\ Procedimiento Clínico}

Una vez realizada la desobturación realizamos la impresión del conducto radicular con resina acrílica Duralay (Reliance, EE.UU) (figura 2 (A)) previo aislamiento del conducto con glicerina, reconstrucción del muñón coronario con resina acrílica Duralay (Reliance, EE.UU) se realizó el tallado del muñón coronario (figura 2(B)) se envió al laboratorio para el colado del poste Cr-Ni (Wironia, Alemania). Para la cementación del poste metálico se realizó la limpieza y secado del conducto radicular y se utilizó cemento autoadhesivo U200 (3M, EE.UU), se retiraron excesos y se polimerizó utilizando la lámpara Valo (Ultradent, EE.UU) a una potencia de $1000 \mathrm{~mW} \mathrm{~cm}{ }^{2}$ por 20 segundos con dirección al poste, para una correcta cementación.

\section{Poste de fibra de vidrio anatomizado Procedimiento Clínico}

Una vez realizada la desobturación se realizó la prueba del poste de fibra de vidrio para corroborar la longitud adecuada, limpieza del poste con ácido fosfórico Scotchbond (3M, EE.UU) por 1 minuto, lavar y secar, con un microbrush se colocó silano Prosil (FGM, Brasil) y adhesivo Single Bond 2 (3M, EE.UU), se polimerizó el adhesivo y se colocó resina Filtek Z350 XT (3M, EE. UU) compuesta alrededor del poste (figura 3(A)) y se introdujo en el conducto radicular para su impresión (figura 3 (B)) previo aislamiento del conducto con glicerina, se polimerizó por 5 segundos y retiró para su evaluación, se volvió a polimerizar por 20 segundos en 2 oportunidades, (40 segundos en total), se reconstruyó el muñón coronario y se realizó la preparación correspondiente (figura 3 (C)). Para la cementación del poste de fibra de vidrio anatomizado, previa limpieza, lavado y secado del conducto radicular, (3M, EE.UU), se utilizó cemento autoadhesivo U200 (3M, EE.UU), se retiraron excesos y se polimerizó utilizando la lámpara Valo (Ultradent, EE.UU) a una potencia de $1000 \mathrm{~mW} \mathrm{~cm}{ }^{2}$ por 20 segundos con dirección al poste, para una correcta cementación. 
Por último, los especímenes fueron enumerados y separados en cada grupo por el investigador, fue asignado un número de orden de manera aleatoria mediante el programa Microsoft Excel 2010 para realizar la prueba compresiva. Cada espécimen fue sometido a una carga compresiva con una velocidad de $1 \mathrm{~mm} / \mathrm{min}$ en una máquina universal de pruebas Instrom, en el Laboratorio High Technology Laboratory Certificate, las muestras fueron desconocidas por el operador de la máquina (un ciego). Para llevar a cabo el test, un dispositivo de acrílico fue fabricado con un espacio en relación con el plano horizontal de $45^{\circ}$ y con una concavidad de forma cuadrada de $2 \times 2$ en el centro, donde los especímenes fueron insertados. Todos los resultados fueron anotaron en la ficha de recolección de datos la cual fue elaborada para la investigación.

El análisis fue realizado mediante una estadística descriptiva que consideró medidas de tendencia

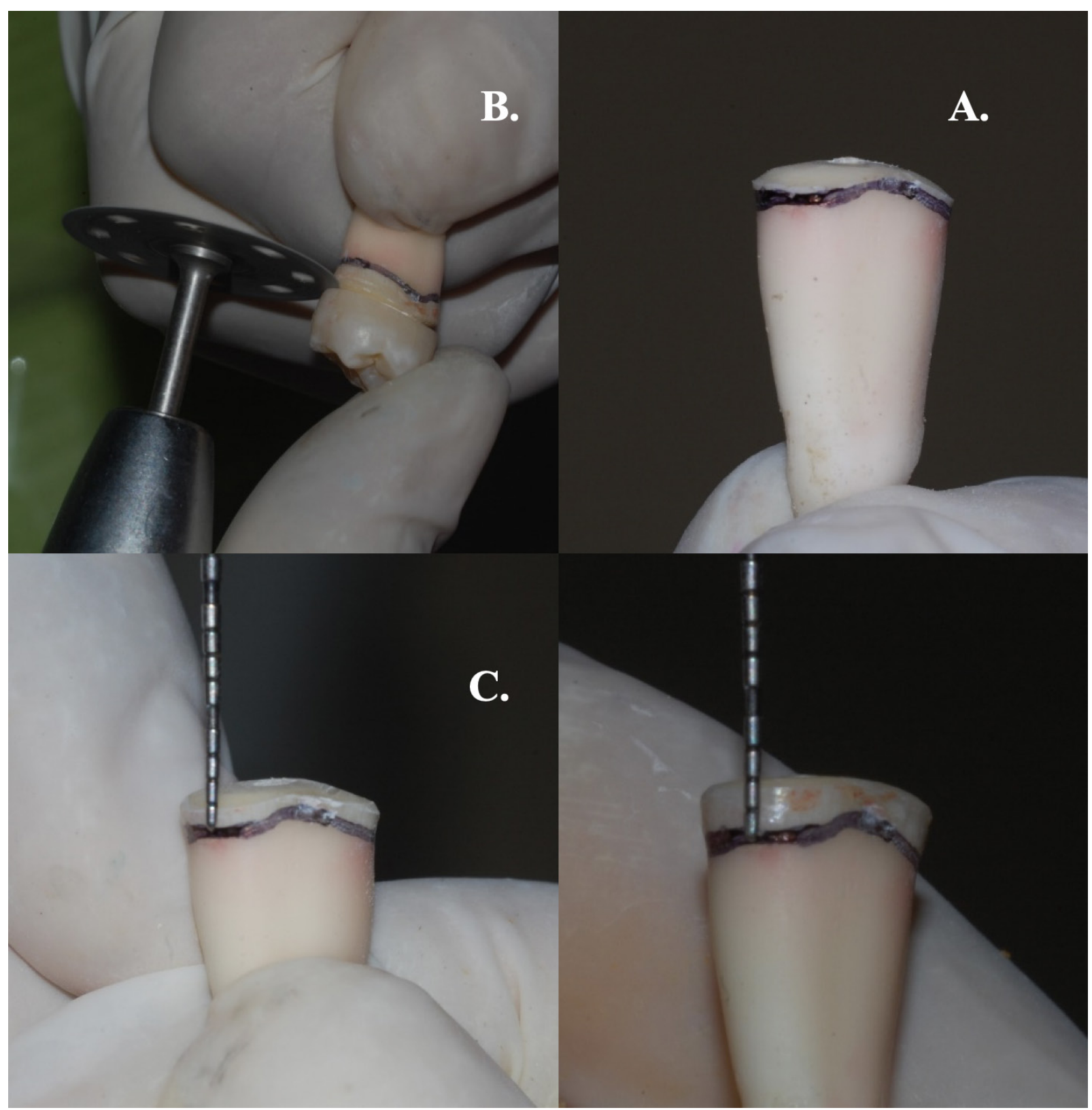

Figura 1. Preparación de las longitudes coronarios. A. Corte de la corona anatómica. B. Longitud coronario de $0 \mathrm{~mm}$ a la unión cemento-esmalte. C. Longitud coronario de $1 \mathrm{~mm}$ a la unión cemento-esmalte. D. Longitud coronario de $2 \mathrm{~mm}$ a la unión cemento-esmalte. 


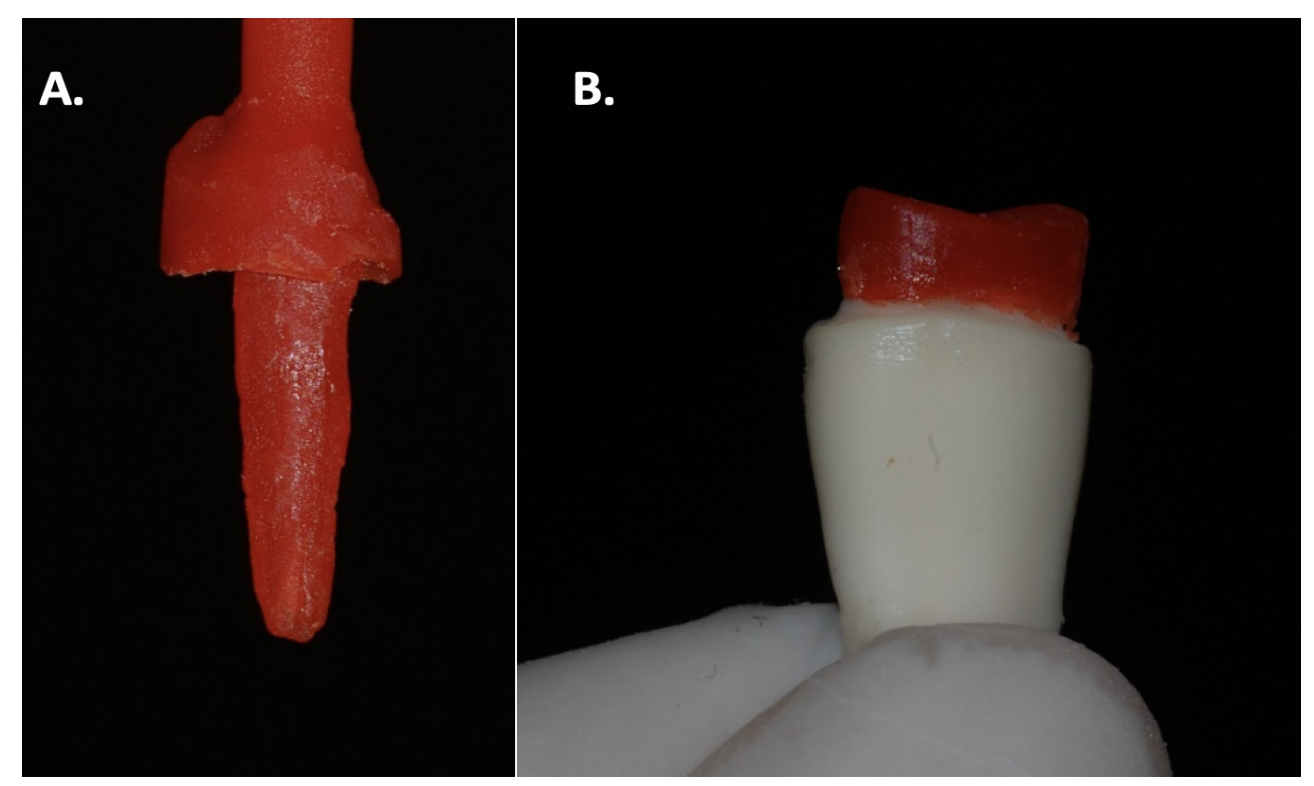

Figura 2. Poste metálico. A. Impresión del conducto radicular con acrílico Duralay. B. Preparación del muñón coronario

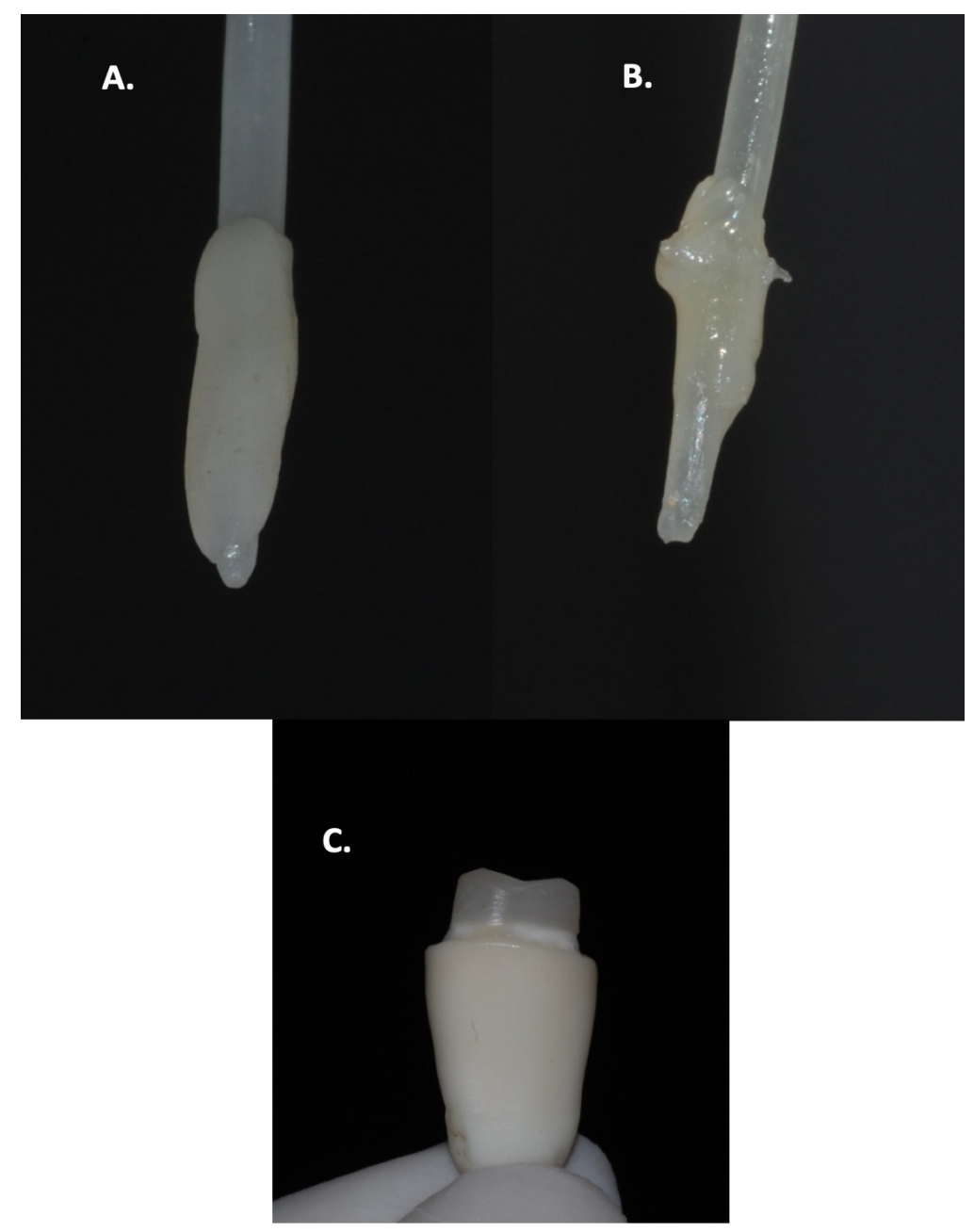

Figura 3. Poste de fibra de vidrio anatomizado. A. Colocación de resina compuesta alrededor del poste. B. Impresión del conducto radicular con resina compuesta. C. Preparación del muñón coronario 
Tabla 1. Resistencia Compresiva del poste metálico según remanente coronario

\begin{tabular}{llllllllll}
\hline & $\mathbf{n}$ & $\mathbf{X}$ & $\mathbf{D E}$ & Min & Max & Rango & $\mathbf{S}^{2}$ & P1 & P2 \\
\hline 0mm & 8 & \multirow{2}{*}{396,05} & 52,07 & 335,98 & 358,98 & 23,98 & 2711,28 & 0,000 entre 0mm y 1 mm \\
PM & & & & & & & & 0,000 entre 0mm y 2 mm \\
$1 \mathrm{~mm}$ & 8 & 531,89 & 109,19 & 782,62 & 782,62 & 458,80 & 11921,84 & 0,001 & $\begin{array}{l}0,000 \text { entre 1 mm y 3 mm } \\
0,000 \text { entre 1 mm y 2 mm }\end{array}$ \\
PM & & & & & & & & & 0,000 entre 2mm y 0 mm \\
2mm & 8 & 885,74 & 104,10 & 1399,05 & 1399,05 & 375,40 & 10835,84 & 0,000 entre 2mm y 1 mm \\
PM & & & & &
\end{tabular}

P1 Anova de un factor

P2 Comparaciones multiples de Tukey

PM Poste Metálico

Tabla 2. Resistencia Compresiva del poste de fibra de vidrio anatomizado según remanente coronario

\begin{tabular}{llllllllll}
\hline & $\mathbf{n}$ & $\mathbf{X}$ & $\mathbf{D E}$ & Min & Max & Rango & $\mathbf{S}^{2}$ & P1 & P2 \\
\hline 0mm & 8 & 462,54 & 52,07 & 431,58 & 495,25 & 63,67 & 2711,28 & 0,118 entre 0mm y 1 mm \\
FV & & & & & & & & 0,000 entre 0mm y 2 mm \\
1mm & 8 & 571,31 & 109,19 & 323,82 & 782,62 & 458,80 & 11921,84 & 0,001 & $\begin{array}{l}0,118 \text { entre 1mm y 3 mm } \\
0,000 \text { entre 1mm y 2 mm }\end{array}$ \\
FV & & & & & & & & & 0,000 entre 2mm y 0 mm \\
2mm & 8 & 962,19 & 104,10 & 783,25 & 1158,65 & 375,40 & 10835,84 & 0,000 entre 2mm y 1 mm \\
FV & & & & & & & & & \\
\hline
\end{tabular}

P1 Anova de un factor

P2 Comparaciones multiples de Tukey

FV Poste de Fibra de Vidrio Anatomizado

central (media y mediana) y medidas de dispersión (desviación estándar, mínimo, máximo, rango y varianza) para la variable estrés compresivo de cada grupo de estudio, se utilizó la prueba de Shapiro Wilk para que se pueda determinar la normalidad, también la prueba ANOVA y por último la de comparaciones múltiples de Tukey con un nivel de significancia de $<0.05$. Todo fue realizado mediante el programa estadístico SPSS versión 20,00.

\section{RESULTADOS}

Esta investigación fue de tipo experimental, después de haber realizado el análisis de la resistencia compresiva, fueron encontrados diversos valores para cada grupo de estudio los cuales fueron anotados en la ficha de recolección de datos.

Se evaluó la existencia o no de diferencias estadísticamente significativas, comparando misma longitud de remanente coronario con diferente material de poste; en el primer grupo de estudio con una longitud de remanente coronario $0 \mathrm{~mm}$ con poste metálico obtuvo un valor promedio de 396,05 (( \pm $52,07)$ mientras que en las piezas dentarias con la misma longitud de remanente coronario $(0 \mathrm{~mm})$, pero con poste de fibra de vidrio anatomizado se obtuvo como valor promedio de 462,54 $( \pm 52,07)$, resultando un mejor comportamiento en los dientes restaurados con poste de fibra de vidrio anatomizado (tabla1 y tabla 2).

También se compararon los dientes con una longitud coronaria de $1 \mathrm{~mm}$ los restaurados con poste metálico obtuvieron un valor promedio de 531,89 ( \pm $109,19)$ y con poste de fibra de vidrio anatomizado, un valor promedio de 571,31 ( $\pm 109,19)$, encontrándose también mayores valores en aquellos restaurados con poste de fibra de vidrio a diferencia de los postes metálicos.

Los dientes con mayor longitud coronaria, $2 \mathrm{~mm}$ restauradas con poste metálico, obtuvieron un valor promedio de $885,74( \pm 104,10)$ y con poste de fibra de vidrio anatomizado, un valor promedio de 962,19 $( \pm 104,10)$ siendo el mayor valor encontrado en éstas comparaciones; $(\mathrm{p}=0,005)$, resultó que existe diferencia estadísticamente significativa entre ambos grupos a diferencia de aquellos dientes con un menor remanente coronario $(0 \mathrm{~mm}$ y $1 \mathrm{~mm})$. 
También se realizaron comparaciones de los dientes con distintos niveles de remanente coronario y el mismo material de poste (fibra de vidrio anatomizado y metálico), lo cual determinó que aquellos dientes con remanentes coronarios de $2 \mathrm{~mm}$ restaurados con poste de fibra de vidrio anatomizado y metálico; presentaron mayores valores de resistencia compresiva a diferencia de los remanentes coronarios de $0 \mathrm{~mm}$ y $1 \mathrm{~mm}$.

Se realizaron comparaciones de los dientes con distintos niveles de remanente coronario y el mismo material de poste; en este caso fibra de vidrio anatomizados; siendo aquellos dientes con remanente coronario de $2 \mathrm{~mm}$ aquellas que presentaron mayor resistencia a la compresión, a diferencia de los dientes con $0 \mathrm{~mm}$ y $1 \mathrm{~mm}$ de remanente coronario.

Así mismo al comparar los dientes con distintas longitudes de remanente coronario pero con poste metálico; también aquellos dientes con remanente coronario de $2 \mathrm{~mm}$ presentaron valores mayores de resistencia compresiva a diferencia de los dientes con $0 \mathrm{~mm}$ y $1 \mathrm{~mm}$ de remanente coronario.

\section{DISCUSIÓN}

Este estudio tiene una importancia clínica debido a que permite entender la importancia superior del nivel de remanente dentario y luego las propiedades mecánicas del material utilizado para el poste. Además, tiene una gran importancia teórica ya que podemos considerarla como un antecedente para próximas investigaciones con la finalidad de contribuir de manera confiable con conocimientos científicos.

Los valores encontrados en este estudio al comparar diversos niveles de remanente coronario con postes de distintos materiales, sirvieron para verificar que independientemente del material utilizado para el poste, es de suma importancia un adecuado remanente coronario para el éxito del tratamiento a lo largo del tiempo.

Varios autores concluyeron en sus estudios que aquellos dientes con un remanente coronario de 2 $\mathrm{mm}$ presenta la más alta resistencia ante la fractura $(15,16,17,18,19,20)$; en nuestro estudio también se concluyó que la existencia de un mayor nivel de remanente coronario $(2 \mathrm{~mm})$ independientemente del material utilizado para la confección del poste presenta mejor desempeño clínico.

Diferentes estudiosevaluaron la resistencia ante la fractura de dientes con tratamientos de conductos, restaurados con postes de fibra de vidrio sobre remanentes coronarios, como resultados se encontró una diferencia estadísticamente significativa entre todos los grupos, aquel grupo con remanente coronario completo, en relación a los otros grupos con remanente coronario incompletos, concluyeron que los dientes que presentaron un nivel de remanente incompleto, presentaron menor resistencia a la fractura $(21,22)$.

Garhnayak et al., y Gbadebo et al., analizaron la distribución de esfuerzos a lo largo de un incisivo central superior con tratamiento de conductos, restaurado con varios sistemas de postes y evaluaron el beneficio del remanente coronario usando el análisis de elementos finitos, la incorporación del nivel de remanente ofreció cierto grado de reducción del estrés en el poste no metálico y aumentó las tensiones dentro de la dentina cervical, en nuestro estudio también se observó que a mayor cantidad de nivel de remanente $(2 \mathrm{~mm})$, se observó el mejor comportamiento biomecánico del poste en el diente con tratamiento de conductos, independientemente del material utilizado $(23,24)$.

Finalmente podemos concluir que el nivel de remanente coronario en postes metálicos y postes de fibra de vidrio anatomizados, restaurados en premolares con tratamientos de conductos, influye en la resistencia compresiva y que los remanentes coronarios de $2 \mathrm{~mm}$ restaurados con poste de fibra de vidrio anatomizado y metálico; presentaron mayores valores de resistencia compresiva a diferencia de los de $0 \mathrm{~mm}$ y $1 \mathrm{~mm}$.

\section{Correspondencia:}

Marcia Edith Vidalón Pinto

Calle Isla Santa Cruz Mz. C1 Lte. 38 Los Cedros de Villa, Zip code 15067.

Chorrillos. Lima, Perú

Correo electrónico: mvidalonp@hotmail.com 


\section{REFERENCIAS BIBLIOGRÁFICAS}

1. Jiang W, YoungChun G, LongXing C. Stress distribution in molars restored with inlays or onlays with or whithout endodontic treatment: A three-dimensional finite element analysis. J Prosthet Dent. 2010; 103 (1): 7-13.

2. Ortega V, Pegoraro L, Conti P, Valle A, Bonfante G. Evaluation of fracture resistance of endodontically treated maxillary premolars, restored with ceromer or heat-pressed ceramic inlays and fixed with dualresin cements. J Oral Rehabil. 2004; 31: 393-7.

3. Koushyar K. Recomendaciones para la selección del material cerámico libre de metal de acuerdo a la ubicación de la restauración en la arcada. Int. J. Odontostomat. 2010; 4; (3): 237-40.

4. Barbosa R, Dias A, Elisaur A, Pereira L, Grassi L. Clinical performance of indirect esthetic inlays and onlays for posterior teeth after 40 months. Braz J. Oral Sci. 2009; 8(3): 154-8.

5. Imanishi, A, Nakamura, T, Ohyama, T, Nakamura, T. 3-D Finite element analysis of all-ceramic posterior crowns. J Oral Rehabil. 2003; 30: 818-22.

6. Yamanel K, Caglar A, Gulsahi K, Ozdem A. Effects of different ceramic and composite materials on stress distribution in inlay and onlay cavities: 3-D finite element analysis. Dent Mater J. 2009; 28 (6): 661-70.

7. Roberson TM, Heymann HO, Swift EJ Jr. Sturdevant's art and science of operative dentistry, 4th ed, USA: Mosby; 2002.

8. Koushyar K. Recomendaciones para la selección el material cerámico libre de metal, de acuerdo a la ubicación de la restauración en la arcada. Int. J. Odontostomat. 2010; 4(3): 237-40.

9. Henry PJ, Bower RC. Post core systems in crown and bridgework. Aust Dent J. 1977; 22: 46-52.

10. Hatzikyriakos AH, Reisis GI, Tsingos N. A 3-year postoperative clinical evaluation of posts and cores beneath existing crowns. J Prosthet Dent. 1992; 67: 454-8.

11. Chandrupatla T. Introducción al Estudio del Elemento Finito en Ingeniería. 2a ed. Prentice may, 1999.

12. Georges AJ, Sturdevant JR, Swift EJ, Thompson JY. Fracture resistance of prepared teeth restored with bonded inlay restoration. J. Prosthet. Dent. 2003; 89 (6): 551-7.

13. Bottino MA, Giannini V, Miyashita E, Quintas AF. Estética en rehabilitación oral: "Metal Free". In: Feller C, Gorab R. Actualización en la clínica odontológica: Módulos de actualización, vol. 1. São Paulo: Artes Médicas, 2000. pp. 325-60
14. Suresh N. Indirect resin composites. J Conserv Dent. 2010; 13: 184-94.

15. Hunter A, Flood A. The restoration of endodontically treated teeth. Part 1. Treatment planning and restorative principles. Aust Dent J. 1988;33:481-490.

16. Zhi-Yue L, Yu-Xing Z. Effects of post-core design and ferrule on fracture resistance of endodontically treated maxillary central incisors. J Prosthet Dent. 2003;89(4):368-73.

17. Bayat MI, Dumbrigue HB, Griggs JA, Wakefield CW. Effect of no ferrule on failure of teeth restored with bonded posts and cores. Gen Dent. 2004;52(2):143-6.

18. Panorjit M, Boonrat S, Boonlert K, Kewalin T. The effect of incomplete crown ferrules on fracture resistance and failure modes of endodontically treated maxillary incisors restored with quartz fiber post, composite core, and crowns. J Conserv Dent. 2015;18(3):187-91

19. Lima AF, Spazzin AO, Galafassi D, Correr-Sobrinho L, Carlini-Júnior B. Influence of ferrule preparation with or without glass fiber post on fracture resistance of endodontically treated teeth. J Appl Oral Sci. 2010;18(4):360-3.

20. Garhnayak L, Parkash H, Sehgal DK, Jain V, Garhnayak M. A comparative study of the stress distribution in different endodonticpost-retainedteeth with and without ferrule design-a finite element analysis. ISRN Dent. 2011;2011:102329. doi: 10.5402/2011/102329

21. Vallego M, Salas A. Fracture resistance of weak teeth with compromised roots rehabilitated with different materials. Rev. CES Odont. 2014; 27(2) 69-80.

22. Gbadebo OS, Ajayi DM, Oyekunle OO, Shaba PO. Randomized clinical study comparing metallic and glass fiber post in restoration of endodontically treated teeth. Indian J Dent Res. 2014;25(1):58-63.

23. Santos-Filho PC, Veríssimo C, Raposo LH, Noritomi PY, Marcondes LR. Influence of ferrule, post system, and length on stress distribution of weakened rootfilled teeth. J Endod. 2014; 40(11):1874-8

24. Sushil K, Arvind T, Chavi T. Effect of different ferrule length on fracture resistance of endodontically treated teeth: An In vitro Study. J Clin Diagn Res. 2017; 11(4):49-52. 